\title{
Metal composition influences optoelectronic quality in mixed-metal lead-tin triiodide perovskite solar absorbers
}

Matthew T. Klug ${ }^{1}$, Rebecca L. Milot ${ }^{1,2}$, Jay B. Patel ${ }^{1}$, Thomas Green ${ }^{1}$, Harry C. Sansom ${ }^{1}$, Michael D. Farrar ${ }^{1}$, Alexandra J. Ramadan ${ }^{1}$, Samuele Martani ${ }^{3}$, Zhiping Wang ${ }^{1}$, Bernard Wenger $^{1}$, James M. Ball ${ }^{1}$, Liam Langshaw ${ }^{1}$, Annamaria Petrozza ${ }^{3}$, Michael B. Johnston ${ }^{1}$, Laura M. Herz ${ }^{1}$, Henry J. Snaith ${ }^{1, *}$

${ }^{1}$ Department of Physics, University of Oxford, Clarendon Laboratory, Parks Road, Oxford OX1 3PU, United Kingdom ${ }^{2}$ Department of Physics, University of Warwick, Gibbet Hill Road, Coventry CV4 7AL, United Kingdom

${ }^{3}$ Center for Nano Science and Technology @Polimi, Istituto Italiano di Tecnologia, Milan, Italy

*email: henry.snaith@physics.ox.ac.uk

Abstract. Current designs for all-perovskite multi-junction solar cells require mixed-metal $\mathrm{Pb}$ $\mathrm{Sn}$ compositions to achieve narrower band gaps than are possible with their neat $\mathrm{Pb}$ counterparts. The lower band gap range achievable with mixed-metal $\mathrm{Pb}-\mathrm{Sn}$ perovskites also encompasses the 1.3 to $1.4 \mathrm{eV}$ range that is theoretically ideal for maximising the efficiency of single-junction devices. Here we examine the optoelectronic quality and photovoltaic performance of the $\left(\left(\mathrm{HC}\left(\mathrm{NH}_{2}\right)_{2}\right)_{0.83} \mathrm{Cs}_{0.17}\right)\left(\mathrm{Pb}_{1-\mathrm{y}} \mathrm{Sn}_{\mathrm{y}}\right) \mathrm{I}_{3}$ family of perovskite materials across the full range of achievable band gaps by substituting between $0.001 \%$ and $70 \%$ of the $\mathrm{Pb}$ content with Sn. We reveal that a compositional range of "defectiveness" exists when Sn comprises between $0.5 \%$ and $20 \%$ of the metal content, but that the optoelectronic quality is restored for Sn content between $30-50 \%$. When only $1 \%$ of $\mathrm{Pb}$ content is replaced by $\mathrm{Sn}$, we find that photoconductivity, photoluminescence lifetime, and photoluminescence quantum efficiency are reduced by at least an order of magnitude, which reveals that a small concentration of Sn incorporation produces trap sites that promote non-radiative recombination in the material and limit photovoltaic performance. While these observations suggest that band gaps between 1.35 and $1.5 \mathrm{eV}$ are unlikely to be useful for optoelectronic applications without countermeasures to improve material quality, highly efficient narrower band gap absorber materials are possible at or below $1.33 \mathrm{eV}$. Through optimising single-junction photovoltaic devices with $\mathrm{Sn}$ compositions of $30 \%$ and $50 \%$, we respectively demonstrate a $17.6 \%$ efficient solar cell with an ideal single-junction band gap of $1.33 \mathrm{eV}$ and an $18.1 \%$ efficient low band gap device suitable for the bottom absorber in all-perovskite multi-junction cells.

Broader Context. Metal-halide perovskite solar cells have many attributes that make them attractive absorber materials for next-generation photovoltaics (PVs). They use earth-abundant elements, are easily processed at low temperatures with scalable deposition techniques, are remarkably defect tolerant, and are highly efficient solar absorbers. In order to contend in an increasingly competitive solar energy marketplace, perovskite-based photovoltaics will need 
M. Klug et al.

\section{Energy \&}

Environmental Science

to offer a lower price-per-watt value than incumbent solar technologies and ultimately a lower levelised cost of electricity for the entire installation. A promising strategy towards this end is to fundamentally increase the overall efficiency of the cell technology by developing multijunction solar cells, which stack perovskite absorbers with different band gaps atop one another. Tremendous progress has been made with tandem solar cells that employ a wide band gap perovskite absorber as the top cell in conjunction with silicon as the bottom cell, which now appear to be poised for high-volume manufacturing. However, this "wafer-based" approach does not fully leverage all the attractive features that the low-temperature processing of thin-film PV provides, such as the ability to deposit directly upon lightweight, flexible substrates, which would enable access to a broader range of solar applications. Yet wholly perovskite multi-junction designs require materials with band gaps below $1.5 \mathrm{eV}$, which are currently only achievable through the partial substitution of $\mathrm{Pb}^{2+}$ ions in the standard perovskite lattice with $\mathrm{Sn}^{2+}$. Development efforts of mixed $\mathrm{Pb}$-Sn perovskites have focused mostly upon the $50-60 \%$ Sn compositions, which yield the lowest band gaps. Yet, relatively little is known about the degree to which these materials can be practically tuned in the broader compositional space. Here, we assess the optoelectronic quality of mixed $\mathrm{Pb}-\mathrm{Sn}$ perovskites across the full band gap range of 1.28-1.55 eV achievable with a formamidinium-caesium lead-tin triiodide perovskite system to determine their suitability for multi-junction and ideal band gap singlejunction photovoltaics.

Introduction. Metal halide perovskites are swiftly approaching a major milestone where they transition from an emergent class of promising solar materials to a competitive power generation technology suitable for large scale deployment. Due to their relative ease of processing, defect tolerance and remarkable optoelectronic properties, perovskites have garnered significant attention from the solar research community over the past seven years, which has produced several methods for reliably processing them into efficient thin-film photovoltaic devices via solution or vapour deposition methods. Now that several perovskite compositions and solar cell architectures routinely exceed $20 \%$ power conversion efficiencies and are demonstrating promising indicators of operational stability, perovskite solar cells are now emerging from the developmental stage and are poised to enter into high-volume manufacturing over the next few years. ${ }^{1-3}$ One of the central attributes of metal halide perovskites that will be key to their successful translation into the energy industry is the tunability of their band gap by chemical composition, which allows them to be optically tailored to specific applications. ${ }^{4}$ Since much of the cost comes from the balance-of-systems, 
M. Klug et al.

\section{Energy \&}

Environmental Science

their ease of processing does not necessarily guarantee less expensive deployed photovoltaic modules. ${ }^{5}$ However, it does provide the opportunity to lower the price-per-watt value by producing multi-junction devices that yield higher power conversion efficiencies with perhaps only marginally higher production cost. The theoretical maximum efficiencies for realistic perovskite-based single-junction, tandem, and triple-junction designs require band gaps spanning 1.22 to $2.04 \mathrm{eV} .{ }^{6}$ For standard lead triiodide perovskite formulations, direct band gaps around 1.5-1.7 eV are achievable using the conventional methylammonium (MA), formamidinium (FA), and caesium (Cs) A-site cations in the $\mathrm{ABX}_{3}$ perovskite lattice. ${ }^{7,8}$ Wider band gaps can be accessed by exchanging some of the iodide content at the X-site with bromide. ${ }^{7,9-12}$ However, the sole route currently available to narrowing the band gap into the near-infrared is through the partial substitution of $\mathrm{Pb}^{2+}$ at the B-site with $\mathrm{Sn}^{2+} \cdot{ }^{13-18}$ For most Asite compositions, band gap bowing occurs in mixed lead-tin $(\mathrm{Pb}: \mathrm{Sn})$ triiodide systems where the band gap narrows continuously with increasing $\mathrm{Sn}^{2+}$ content until about 50-80\% where it reaches a minimum and then proceeds to widen thereafter with further $\mathrm{Sn}^{2+}$ content. ${ }^{13,19-23}$ To date, much of the research effort in $\mathrm{Pb}: \mathrm{Sn}$ blends has focused on compositions that produce the lowest band gaps in order to develop a bottom cell absorber for perovskite-perovskite tandems. Compositions of $\mathrm{MA}\left(\mathrm{Pb}_{0.5} \mathrm{Sn}_{0.5}\right) \mathrm{I}_{3}$, $\left(\mathrm{FA}_{0.5} \mathrm{MA}_{0.5}\right)\left(\mathrm{Pb}_{0.5} \mathrm{Sn}_{0.5}\right) \mathrm{I}_{3}, \quad\left(\mathrm{FA}_{0.6} \mathrm{MA}_{0.4}\right)\left(\mathrm{Pb}_{0.4} \mathrm{Sn}_{0.6}\right) \mathrm{I}_{3}$, $\left(\mathrm{FA}_{0.7} \mathrm{MA}_{0.3}\right)\left(\mathrm{Pb}_{0.5} \mathrm{Sn}_{0.5}\right) \mathrm{I}_{3}$, $\left(\mathrm{FA}_{0.75} \mathrm{Cs}_{0.25}\right)\left(\mathrm{Pb}_{0.5} \mathrm{Sn}_{0.5}\right) \mathrm{I}_{3}$, and $\left(\mathrm{Cs}_{0.025} \mathrm{FA}_{0.475} \mathrm{MA}_{0.5}\right)\left(\mathrm{Pb}_{0.5} \mathrm{Sn}_{0.5}\right) \mathrm{I}_{3}$ have all yielded power conversion efficiencies exceeding $15 \%$ in single-junction solar cells $^{14,15,19,23-27}$ with FA:Cs, FA:MA, and Cs:FA:MA compositions recently reaching efficiencies exceeding 20\% ${ }^{26-29}$ However, the development of compositions with band gaps other than the narrowest possible has been relatively less explored even though several other strategic band gaps are accessible through $\mathrm{Pb}: \mathrm{Sn}$ blending, such as $1.33 \mathrm{eV}$ and $1.44 \mathrm{eV}$, which are respectively ideal for single-junction devices and the middle junction in triple-junction perovskite-perovskite-silicon solar cells. ${ }^{6}$ The incorporation of $\mathrm{Sn}^{2+}$ into metal halide perovskites is seemingly straight-forward since it is one of the few metal species capable of definitively occupying the B-site of the lattice ${ }^{30}$, yet complications arise from its tendency to oxidise to $\mathrm{Sn}^{4+} .16,31,32$ It has been reported that $\mathrm{Pb}: \mathrm{Sn}$ blends degrade via a different mechanism than neat $\mathrm{Sn}$ perovskites since the presence of neighbouring $\mathrm{Pb}^{2+}$ ions modifies the activation energy of $\mathrm{Sn}^{2+}$ oxidation. ${ }^{33}$ However, the exclusion of $\mathrm{Sn}^{4+}$ from the perovskite lattice inevitably produces tin vacancies during the initial film crystallisation ${ }^{31,34}$ and upon later film degradation ${ }^{33}$, which leads to $\mathrm{p}$-doping of the perovskite film and reduced charge carrier lifetimes. Strategies have been developed to cope with tin vacancy formation, such as adding reducing agents, ${ }^{35,36}$ the comproportionation of $\mathrm{Sn}(0)$ and $\mathrm{Sn}(\mathrm{IV})$ to $\mathrm{Sn}(\mathrm{II}),{ }^{27}$ or using an excess 
M. Klug et al.

\section{Energy \&}

Environmental Science

of $\mathrm{Sn}$ (II) salts, with $\mathrm{SnF}_{2}$ being the most common. ${ }^{37,38}$ Yet even champion low band gap $\mathrm{Pb}: \mathrm{Sn}$ perovskites suffer from photoluminescence lifetimes and quantum efficiencies substantially lower than their neat $\mathrm{Pb}$ counterparts, ${ }^{15,20,39}$ although additives such as guanidinium thiocyanate $^{28}$ and guanidinium bromide ${ }^{40}$ have recently yielded improvements. A deeper investigation of how Sn composition impacts optoelectronic quality and the identification of potential sources of defectiveness is required to develop countermeasures capable of improving the performance and stability of $\mathrm{Pb}: \mathrm{Sn}$ perovskite devices.

To evaluate how partial lead replacement by tin influences the optoelectronic quality of metal halide perovskites, we prepared and characterised perovskite thin-films with Sn comprising between $0.001 \%$ and $70 \%$ of the nominal metal content. A blended composition of $\mathrm{FA}_{0.83} \mathrm{Cs}_{0.17}$ was selected for the A-site because it has proven to yield a crystallographically and thermally stable three-dimensional triiodide perovskite phase with neat $\mathrm{Pb} .{ }^{41}$ Furthermore, FA:Cs blends with a Sn content of $50 \%$ have demonstrated superior long-term device stability compared with MA analogues, which makes it a very relevant candidate for practical integration into photovoltaic devices. ${ }^{42}$ By characterising both thin-films and full photovoltaic devices, we assess the optical, electronic, and crystallographic properties as a function of Sn content and correlate them with indicators of defectiveness. For the first time, we find Sn substitution can have a detrimental impact on the perovskite when as little as $0.1 \%$ of $\mathrm{Pb}^{2+}$ is replaced, which reveals that tin incorporation is introducing defects that deteriorate photovoltaic performance. Compared to the neat $\mathrm{Pb}$ composition, a nominal $\mathrm{Sn}$ content of $1 \%$ doubles both the Urbach energy and non-radiative voltage loss in devices, yields an order of magnitude reduction in photoconductivity, more than halves device efficiency, and quenches both photoluminescence and electroluminescence. We identify a compositional range of $0.5-20 \% \mathrm{Sn}$ content in the $\left(\mathrm{FA}_{0.83} \mathrm{Cs}_{0.17}\right)\left(\mathrm{Pb}_{1-\mathrm{y}} \mathrm{Sn}_{\mathrm{y}}\right) \mathrm{I}_{3}$ series as being electronically defective, where optoelectronic quality and photovoltaic performance are reduced. However, when the Sn content reaches and exceeds $30 \%$, we find that material quality is restored, and photovoltaic performance reaches power conversion efficiencies over $15 \%$ for $50 \% \mathrm{Sn}$. By optimising solar cells with $30 \% \mathrm{Sn}$, we achieve a $1.33 \mathrm{eV}$ perovskite solar cell that displays a phase-pure X-ray diffraction pattern and a champion steady-state power output of $17.6 \%$. Similarly, with $50 \%$ Sn we achieve a $1.30 \mathrm{eV}$ band gap device with a steady-state efficiency of $18.0 \%$. Our findings demonstrate that the optoelectronic properties of $\left(\mathrm{FA}_{0.83} \mathrm{Cs}_{0.17}\right)\left(\mathrm{Pb}_{1-\mathrm{y}} \mathrm{Sn}_{\mathrm{y}}\right) \mathrm{I}_{3}$ are limited by inherent defects introduced by $\mathrm{Sn}$ itself when it comprises between $0.5 \%$ and $20 \%$ of the metal content, which hinders photovoltaic performance in 1.35-1.5 eV band gap materials. 
M. Klug et al.

\section{Energy \&}

Environmental Science

Results and Discussion. Thin-films of $\left(\mathrm{FA}_{0.83} \mathrm{Cs}_{0.17}\right)\left(\mathrm{Pb}_{1-\mathrm{y}} \mathrm{Sn}_{\mathrm{y}}\right) \mathrm{I}_{3}$ were deposited using the antisolvent quenching method during spin-coating and the exact same set of fabrication parameters were used for all $\mathrm{Pb}: \mathrm{Sn}$ compositions. The metal content of each composition is defined by the molar ratio of $\mathrm{PbI}_{2}: \mathrm{SnI}_{2}$ in the precursor solution, which also contains a $10 \%$ molar excess of $\mathrm{SnF}_{2}$ over the $\mathrm{SnI}_{2}$ content and a $6 \%$ molar excess of $\mathrm{Pb}(\mathrm{SCN})_{2}$ over the $\mathrm{PbI}_{2}$ content. The addition of $\mathrm{SnF}_{2}$ is a common practice in Sn-rich perovskites, which is thought to decrease the formation of tin vacancies and reduce the background hole density in the material, which would otherwise lower the minority carrier (electron) lifetime. ${ }^{31,32}$ Likewise, $\mathrm{Pb}(\mathrm{SCN})_{2}$ has been reported to be a helpful additive that improves performance in both neat $\mathrm{Pb}$ and $\mathrm{Pb}: \mathrm{Sn}$ compositions. ${ }^{14,15,43-45}$ This blend of $\mathrm{FA}^{+}$and $\mathrm{Cs}^{+}$at the A-site allows thin-films of each $\mathrm{Pb}: \mathrm{Sn}$ composition to nucleate directly into the black perovskite phase upon dispensing the antisolvent at room-temperature and a 30 minute anneal at $120^{\circ} \mathrm{C}$. Scanning electron microscopy (SEM) reveals that this method produces films that are virtually pinhole-free for every $\mathrm{Pb}: \mathrm{Sn}$ composition investigated (Figure S1). We assessed the optical properties of each $\mathrm{Pb}: \mathrm{Sn}$ composition using UV-Vis-NIR spectrophotometry and steady-state photoluminescence (PL). We report the absorption and PL emission spectra in Figure S2 and extract the optical band gap for each composition using both a Tauc plot of absorption (Figure S3a) and the PL peak position. Although band gap values extracted from Tauc plots and PL peaks are commonly used to specify the band gap of a material and produce lower values, in this work we have chosen to derive the band gap value from the peak of the first derivative of the external quantum efficiency (EQE) spectrum (Figure S4a) as suggested by Rau et al, which is considered a more physically relevant parameter for solar cells. ${ }^{46,47}$ For comparison, we present the band gap we determined with each of these three methods in Figure 1a. Independent of which measure is used to define the band gap, we observe the expected band gap narrowing and bowing behaviours for this compositional series with the lowest value occurring with $60 \%$ Sn content, which is consistent with previous reports for similar systems. ${ }^{14,19,20,23}$

From powder X-ray diffraction measurements (XRD) on thin-films, we reveal that a polycrystalline material is produced for each $\mathrm{Pb}: \mathrm{Sn}$ composition (Figure S5) and we show each of the three main perovskite diffraction peaks in Figure 1c-e. We determined the lattice volume for each $\mathrm{Pb}: \mathrm{Sn}$ composition through Pawley fits of the powder XRD patterns to an orthorhombic unit cell in the Pnam space group, and reveal in Figure $1 \mathrm{~b}$ a contraction of the lattice with increasing $\mathrm{Sn}$ content, consistent with the smaller sized $\mathrm{Sn}^{2+}$ cation ${ }^{48}$ occupying an increasing larger percentage of the B-sites in the perovskite lattice. 
M. Klug et al.

\section{Energy \&}

Environmental Science

We fabricated solar cells with the p-i-n device architecture, using phenyl-C61-butyric acid methyl ester (PCBM) as the n-type contact and a bilayer polymer stack of poly[bis(4phenyl)(2,4,6-trimethylphenyl)amine]

and

poly $\left[\left(9,9-b i s\left(3^{\prime}-(\mathrm{N}, \mathrm{N}-\right.\right.\right.$ dimethylamino)propyl)-2,7-fluorene)-alt-2,7-(9,9-dioctylfluorene)] (PFN) as the p-type contact. Modifying hydrophobic hole transporters such as PTAA with a thin layer of PFN improves the wettability of the perovskite solution, increases device reproducibility, and suppresses interfacial recombination. ${ }^{49,50}$ We show the full device architecture in Figure 2a. In order to accurately measure solar cell performance across the full range of band gaps, we calibrated the solar simulator independently for the $0 \%$ and $50 \% \mathrm{Sn}$ compositions and all efficiencies were carefully corrected for spectral mismatch using the EQE spectra (see Figure S6-S10, Table S1, and the Supporting Information for more discussion). ${ }^{51}$ Box plots of the steady-state power output (SPO) and each photovoltaic performance metric are shown for the examined metal compositions in Figure $2 b-f$. The current-voltage characteristics and SPO time traces for champion devices from each composition are shown in Figure S11-S12, boxplots with individual data points for the forward and reverse scans are shown in Figure S13, and the champion and mean performance metrics are tabulated in Table S2. We observe that both the wide $(0 \% \mathrm{Sn})$ and narrow $(50 \% \mathrm{Sn})$ band gap compositions are capable of yielding power conversion efficiencies (PCE) and SPOs above 15\% with this device architecture.

As the $\mathrm{Sn}$ content in the perovskite is increased with respect to the neat- $\mathrm{Pb}$ composition and the band gap narrows, we expect the short-circuit current density $\left(\mathrm{J}_{\mathrm{sc}}\right)$ to increase and the opencircuit voltage $\left(\mathrm{V}_{\mathrm{oc}}\right)$ to decrease. However, we find that both of these metrics drop considerably when Sn comprises between $0.5 \%$ and $20 \%$ of the nominal metal content, which result in decreased PCE within this compositional range.

In order to understand why compositions within this range do not operate very effectively in solar cells, we firstly consider charge extraction. Theoretical investigations have shown that $\mathrm{Sn}$ addition in mixed-metal $\mathrm{Pb}: \mathrm{Sn}$ perovskites narrows the band gap by raising the valence band maximum closer to vacuum level through adding new states to the band edge. ${ }^{52}$ Therefore, we expect that if good charge extraction is achieved at the charge selective contacts for both the widest and narrowest band gaps, it should also be efficient for any $\mathrm{Pb}: \mathrm{Sn}$ composition with intermediate values. To probe this experimentally, we performed X-ray photoemission spectroscopy measurements (Figure S14) on select Sn compositions to determine how Sn content impacts the position of the valence band maximum relative to the Fermi level. Assuming the Fermi level resides at $0 \mathrm{eV}$, Figure $\mathrm{S} 14 \mathrm{a}$ shows a clear monotonic shifting in the 
M. Klug et al.

position of the valence band towards the Fermi level when $\mathrm{Sn} \geq 1 \%$, which suggests that energetic changes are likely continuous between the widest and narrowest band gap compositions. We cannot state from these measurements alone if the shift is due to the valence band moving closer to the vacuum level, or due to the Fermi level moving closer to the valence band maximum (i.e. the material becoming more p-type). However, the absence of discernible "S-kinks" in the current-voltage characteristics for each composition (Figure S11) indicates that any energetic barriers at the interfaces either do not exist or are small enough to not impede charge collection.

Turning our attention to the absorber layer itself, we first assessed the impact of $\mathrm{Pb}(\mathrm{SCN})_{2}$ addition upon the material and photovoltaic properties of the perovskite series. Consistent with previous findings ${ }^{45}$, its inclusion in the precursor solution generates both $\mathrm{PbI}_{2}$ and $\delta$ - $-\mathrm{CsPI}_{3}$ impurities phases when $\mathrm{Sn} \leq 20 \%$ (Figure S5). However, by fabricating a series of devices without any $\mathrm{Pb}(\mathrm{SCN})_{2}$, we demonstrate that the observed reductions in solar cell performance are not due to the additive and the associated impurities, but rather result from the incorporation of Sn itself into the perovskite film (see Figure S15-S21, Table S3, and Supporting Information for further discussion). We then performed a series of optoelectronic and material characterisation techniques across the compositional range in order to investigate why $\mathrm{Sn}$ is detrimental to photovoltaic performance in certain compositions. We measured the absorption spectra of perovskite thin-films with select Sn compositions using the highly sensitive technique of photothermal deflection spectroscopy (PDS), which provides direct measures of both sub-bandgap absorption and absorption at the band edge. As we show in Figure 3a, the sub-bandgap absorbance values are lowest for the neat $\mathrm{Pb}$ and $0.01 \% \mathrm{Sn}$ compositions but begin to increase at $0.1 \% \mathrm{Sn}$, which generally continues until the highest measured subbandgap absorbance occurs for $50 \% \mathrm{Sn}$. We note that PDS is not sensitive to optical scattering and these measurements are well within the detection limit of the apparatus, and hence represent unambiguous sub-bandgap absorption. This monotonic trend in sub-bandgap absorption with increasing Sn content may be explained by increased free carrier absorption due to electronic hole doping. The chemical susceptibility of $\mathrm{Sn}^{2+}$ cations to oxidation means that inevitably some of the population will oxidise to $\mathrm{Sn}^{4+}$ and leave B-site vacancies as they are excluded from the perovskite lattice. To resolve this apparent violation of charge neutrality, two holes are released from the valence band to compensate for each B-site vacancy, and the energy level of the vacancies is such that the holes are not trapped but induce p-doping in the absorber material. ${ }^{20,53}$ In other words, the tin vacancy is a shallow acceptor state near the 
M. Klug et al.

\section{Energy \&}

Environmental Science

valence band edge. It has been proposed that the formation energy of tin vacancies is low in neat $\mathrm{Sn}$ materials, but increases in mixed $\mathrm{Pb}: \mathrm{Sn}$ perovskites since the presence of $\mathrm{Pb}^{2+}$ in neighbouring lattice B-sites raises the energy required to break metal-halide-metal bonds. ${ }^{33}$ By this argument, the perovskite should become increasingly prone to p-doping and subsequently higher sub-bandgap absorption by these free carriers with increasing Sn content, which is consistent with observations of p-type character in low-bandgap perovskites, and also consistent with our XPS measurements here. ${ }^{19,54}$

If the free carrier density is increased by p-doping and the charge carrier mobility is invariant with changes in composition, we would expect to observe an increase in the conductivity of the film. In order to probe conductivity, we performed four-point probe measurements under both illuminated and dark conditions. Figure $3 \mathrm{~b}$ shows that the perovskite film conductivity in the dark is initially very low, $10^{-7} \mathrm{~S} / \mathrm{cm}$, with the neat $\mathrm{Pb}$ compound and exhibits a gradual monotonic decrease with increasing Sn content to a minimum approaching $\sim 10^{-8} \mathrm{~S} / \mathrm{cm}$ at a Sn content near 5\%. However, when the Sn fraction is increased beyond $20 \%$, we observe a very steep monotonic increase in conductivity, reaching values over $10^{-2} \mathrm{~S} / \mathrm{cm}$ for $70 \% \mathrm{Sn}$. We observe a similar trend when we measure the conductivity under light, however the initial drop in light conductivity from $0 \%$ to $5 \% \mathrm{Sn}$ is over two orders of magnitude.

Conductivity is a product of charge carrier mobility and charge carrier density. Therefore, the observed compositional dependence in conductivity could arise from changes in either or both of these quantities. In order to assess how charge carrier mobility varies over the compositional range, we employed the contact-free optical-pump-terahertz-probe (OPTP) photoconductivity technique. We observe some variations in the short-range mobilities in Figure 3c, but there is a general trend of increasing mobility with increasing Sn-fraction. This is consistent with the reduced atomic mass of $\mathrm{Sn}^{2+}$, compared with $\mathrm{Pb}^{2+}$, increasing the longitudinal optical phonon frequencies, and in turn, reducing the strength of Fröhlich electron-phonon interactions. ${ }^{32,55}$ There are some variations in mobility values with the lowest being $26 \mathrm{~cm}^{2} / \mathrm{Vs}$ with $1 \% \mathrm{Sn}$ and another apparent drop at 40\% Sn. However, the relative differences between the local extrema are small and all these values are consistent with high-quality perovskite materials over the "short-range". To place this in context, we determined the OPTP mobility to be only $2 \mathrm{~cm}^{2} / \mathrm{Vs}$ in our first report of $\mathrm{MASnI}_{3}$, which delivered a hero solar cell power conversion efficiency of only $6 \% .{ }^{16}$ Likewise, in disordered Br-rich lead mixed-halide compositions, the mobility can be as low as $\sim 5 \mathrm{~cm}^{2} / \mathrm{Vs}{ }^{56,57}$ Notably, the charge carrier mobilities of metal halide perovskites are inherently limited to the order of $100 \mathrm{~cm}^{2} / \mathrm{Vs}$ by phonon-scattering, which means that unlike 
M. Klug et al.

\section{Energy \&}

Environmental Science

long-range four-point probe conductivity measurements, the OPTP technique is only sensitive to scattering on very short length-scales, about a few nanometres. In these examples of lower mobility "defective materials", the interpretation with OPTP has been that there must be additional charge carrier scattering from structural defects within the perovskite grains. The fact that we do not observe a mobility reduction within our "defective" compositional zone by OPTP suggests that short-range crystalline order and quality in these materials is very high.

Returning to the four-point probe conductivity measurements, the drop in dark conductivity with increasing Sn content up to $20 \%$, despite an increase in background p-doping (which we infer from the increased sub-bandgap absorption), suggests that there is a decrease in the longrange charge carrier mobility with increasing Sn content over this range. The more significant drop in the light conductivity over the same range, also suggests that there is a reduction in the steady-state photogenerated carrier density, which would result from a reduction in the carrier lifetime.

In order to assess how the radiative and non-radiative recombination vary with changing $\mathrm{Sn}$ content, we employed photoluminescence (PL) spectroscopy. We determined the PL quantum efficiency (PLQE) near 1 sun equivalent irradiance (Figure 3d, excitation with $532 \mathrm{~nm}$ laser) and PL lifetimes (Figure 3e) using steady-state and time-resolved techniques, respectively. We fit the PL decay profiles with a stretched exponential model with lifetimes defined as the time for the signal to reduce by a factor of 1/e from its initial value (see Supporting Information). For the neat $\mathrm{Pb}$ composition, we find that the perovskite material is strongly emissive with PLQEs reaching over $14 \%$ at $115 \mathrm{~mW} / \mathrm{cm}^{2}$ irradiance and a PL lifetime of $1.1 \mu \mathrm{s}$. When the metal content is only $0.001 \% \mathrm{Sn}$, we observe a clean PL signal (Figure S22b) that yields the highest PLQE, which peaks at over $15 \%$, and the longest PL lifetime of $1.4 \mu \mathrm{s}$. With further increases of Sn content up to $\sim 0.01 \% \mathrm{Sn}$, the PLQE and PL lifetime remain similar to the neat $\mathrm{Pb}$ perovskite, which is relatively encouraging since it confirms that a reasonably large amount of Sn "contamination" will not be detrimental to the Pb-based perovskites. However, for the 0.5-5\% Sn compositions, we observe considerable quenching of the PL emission, thereby rendering the PLQE below our detection threshold of $0.1 \%$ and reducing the PL lifetimes by two orders of magnitude to about 10 ns (Table S4). It must be noted that the PL peak position is constant within this compositional range (Figure S22a), which shows that the dramatic reductions in emission with $\mathrm{Sn}$ content is not due to any changes in the material band gap. Unintentional p-doping is expected to produce low PL lifetimes because there is a background sea of holes released from dopant sites that are available to recombine with photogenerated 
M. Klug et al.

\section{Energy \&}

Environmental Science

electrons in the conduction band. ${ }^{20,32,53}$ However, it has been shown in Sn-rich perovskites that this pseudo-monomolecular recombination pathway is radiative and can produce PLQE gains ${ }^{20}$ as well as amplified spontaneous emission in the case of FASnI $3 .{ }^{53}$ Therefore, what we are observing in this compositional range is a significant increase in non-radiative recombination, which is therefore not due to the increased background density of holes, but rather to new defects responsible for trap-assisted recombination.

Examination of the PDS absorption edges in Figure 3a reveals a linear exponential character of the band tails, which allows for a measure of disorder to be quantified for each perovskite composition in the form of the Urbach energy, $E_{U}$ (Figure 3f)..$^{58,59}$ These values are less than $19 \mathrm{meV}$ when $\mathrm{Sn}$ is below $0.1 \%$ but increases thereafter with Sn content, reaching up to 42 $\mathrm{meV}$ for $50 \% \mathrm{Sn}$. This increase is significant, even without increased non-radiative recombination, since the theoretical open-circuit voltage in the radiative limit becomes substantially reduced due to increased dark recombination current when $\mathrm{E}_{\mathrm{U}}>\mathrm{kT}$. ${ }^{60}$ While this trend is consistent with PDS measurements of MA-based Pb:Sn analogues ${ }^{39}$, we find that the Urbach energies extracted from PDS are not independent of free carrier absorption. Figure S23 shows that when a uniform "panchromatic" background is added to the absorbance spectrum for the $0 \%$ Sn sample in order to bring the sub-bandgap absorption to the level measured for the $50 \%$ Sn sample, the extracted $E_{U}$ rises by more than $50 \%$ from 18.9 to $29.1 \mathrm{meV}$. This apparent change has evidentially nothing to do with a change in the continuum of states within the absorber but is a mathematical artefact that arises from adding a constant offset to an exponentially rising function. While PDS measurements provide a useful means to sensitively probe sub-band gap absorptions, the Urbach energies extracted from this technique might not be the most appropriate measure of optoelectronic quality in doped perovskite systems because free carrier absorption can obfuscate their interpretation.

A complementary optoelectronic technique that also offers high enough sensitivity and dynamic range to characterise the Urbach band tail is Fourier-transform photocurrent spectroscopy (FTPS). Whereas PDS measures all absorption events, FTPS measures only those that lead to photocurrent generation. By sampling the band tail with FTPS, Urbach energies can be extracted from EQE measurements that are isolated from free carrier absorption and provide a more relevant metric of disorder in our unintentionally p-doped $\mathrm{Pb}: \mathrm{Sn}$ perovskites (Figure S24). In contrast to the PDS measurements, we find that the Urbach energies measured through FTPS do not increase monotonically with Sn content (Figure 3f), rather Eu values are initially low (14-15 meV) when $\mathrm{Sn}<0.5 \%$, double to around $30 \mathrm{meV}$ near $5 \% \mathrm{Sn}$, and then 
M. Klug et al.

\section{Energy \&}

Environmental Science

gradually return to $14-15 \mathrm{meV}$ and remain there when $\mathrm{Sn}>50 \%$. This zone of increased Urbach energies between $0.5 \%$ and $20 \% \mathrm{Sn}$ content corresponds precisely to the compositional range where we observed significant reductions in photovoltaic efficiency (Figure 2) and long-range conductivity (Figure 3b), whereas the widest and narrowest gap compositions display a comparatively ordered nature. By definition, the difference between the theoretical maximum $\mathrm{V}_{\mathrm{oc}}$ for a given $\mathrm{E}_{\mathrm{g}}$ and the value measured in actual devices is the voltage loss due to nonradiative recombination, $\Delta V^{N R}$, which provides a measure of photovoltaic defectiveness that is decoupled from band gap. ${ }^{61-63}$ In our perovskite system, Figure $3 g$ shows a $\Delta V^{N R}$ around 250 $\mathrm{mV}$ is achieved near the neat $\mathrm{Pb}(0-0.1 \% \mathrm{Sn})$ and low band gap $(30-50 \% \mathrm{Sn})$ compositions, but the intermediate range has substantially increased values that peak at 5\% Sn content with 520 $\mathrm{mV}$ non-radiative loss. While the Urbach energies measured by FTPS are too low to produce significant voltage reductions from the Shockley-Quiesser limit due to radiative recombination at tail states ${ }^{47,60}$, there remains a clear correlation between $\Delta V^{N R}$ and $\mathrm{E}_{\mathrm{U}}$ as measured by FTPS (Figure 3f). It is therefore consistent that the electronic disorder we observe at the band edge for this intermediate $\mathrm{Sn}$ composition regime is symptomatic of the presence of defects responsible for trap-assisted non-radiative recombination.

To summarise our findings, all of these measures of optoelectronic quality (i.e., power conversion efficiency, non-radiative voltage loss, Urbach energy, conductivity, PL lifetimes, and PLQE) suggest that the $\mathrm{Pb}: \mathrm{Sn}$ compositional range investigated here for $\left(\mathrm{FA}_{0.83} \mathrm{Cs}_{0.17}\right)-$ based triiodide perovskites can be divided into three separate zones. (1) Neat Pb perturbation $(\mathrm{Sn}<0.5 \%)$ : When the $\mathrm{Sn}$ content is $0.1 \%$ or less, the perovskite exhibits high optoelectronic quality characterised by long PL lifetimes, high PLQE, low non-radiative voltage loss, and low Urbach energies near $15 \mathrm{meV}$ from FTPS and $18 \mathrm{meV}$ from PDS. Only beyond around $0.1 \%$ Sn content is there an observed increase in sub-bandgap free carrier absorption, which we attribute to the onset of p-doping via Sn-vacancies at some B-sites. The band gap is invariant in this compositional range (Figure 1a), whereas the crystal lattice undergoes expansion with Sn content (Figure 1b), which accompanies a reduction in lattice microstrain (Figure 4a). (2) Defective zone $(0.5 \% \leq \mathrm{Sn} \leq 20 \%)$ : After $0.1 \% \mathrm{Sn}$, the microstrain rises to a peak value at $0.5 \%$ $\mathrm{Sn}$ and the material enters a phase of rapid crystallographic and electronic change. The lattice volume contracts from $1023 \AA^{3}$ at $0.1 \%$ to $1004 \AA^{3}$ at $20 \%$ Sn (Figure 1b) as the band gap accordingly reduces from 1.55 to $1.4 \mathrm{eV}$ (Figure 1a). In this range, free carrier absorption continues to rise with $\mathrm{Sn}$ content and the perovskite material itself exhibits defectiveness consistent with a reduction in carrier lifetime and increased non-radiative recombination. One 
possible explanation is that crystallographic disorder is introduced as the lattice contracts not only because of local strain caused by the undersized $\mathrm{Sn}^{2+}$ ions and $\mathrm{Sn}$-vacancies at the B-sites but also because contraction can proceed either isotropically or through octahedral tilting. ${ }^{23}$ It is possible that different spatial regions within the poly-crystalline thin-film experience each of these crystallographic distortions to varying degrees or there is local heterogeneity in $\mathrm{Sn}$ content, which could lead to energetic disorder at the band edge and potentially accommodate deeper trap formation. This structural disorder is compounded by the fact that this is also the most rapid region of band gap change, which is demonstrated in Figure 4a by the compositional derivative of the band gap peaking around $10 \% \mathrm{Sn}$. Changes in the bond angles of metalhalide-metal bonds of perovskites will alter the degree of molecular orbital overlap and induce changes to the local electronic band structure. ${ }^{64,65}$ Yet when the band gap changes rapidly with composition, it is expected that even minute, yet significant amounts of crystallographic disorder will lead to greater than usual variation in the electronic band structure, and hence amplify the degree of electronic defectiveness within the bulk perovskite thin-film. This is supported by broadening in the PL and EQE derivative peaks largely within this compositional zone (Figure 4b, Figure S25 and Figure S26). To illustrate this notion, we multiplied the measured microstrain (Figure 4a), which quantifies the non-uniform local strain within the perovskite and indicates structural disorder (see Figure S27 for analysis), by the compositional derivative of the band gap, which indicates the sensitivity of the perovskite electronic band structure to metal composition, and find that the shape of the product (Figure 4c) correlates well with the FTPS Urbach energy and non-radiative voltage loss trends presented in Figure 3. (3) Low band gap plateau $(20 \%<\mathrm{Sn} \leq 70 \%)$ : Once the nominal Sn content surpasses $20 \%$, the perovskite enters a more crystallographically and electronically stable compositional region where the lattice volume ceases to contract (Figure 1b), the microstrain remains relatively low (Figure 4a), and the rate of change of the band gap slows appreciably as it approaches the minimum at $60 \%$ Sn (Figure 4a). Remarkably, the material regains a state of good optoelectronic quality, which is indicated by both the non-radiative voltage loss reaching the lowest values for the whole $\mathrm{Pb}: \mathrm{Sn}$ compositional series (Figure 3g) and FTPS Urbach energies similar to those found in the neat $\mathrm{Pb}$ perovskites (Figure 3f). Similarly, it is around 30\% Sn content that light conductivities return to values obtained by the neat $\mathrm{Pb}$ perovskite composition (Figure 3b) and dark conductivities rise, which indicates hole doping occurs concomitantly with an increase in long-range charge carrier mobility and a reduction in non-radiative recombination. Photovoltaic performance peaks at 50\% Sn composition and falls thereafter. 
M. Klug et al.

\section{Energy \&}

Environmental Science

Beyond 50\% Sn, high performing devices are more difficult to reproduce, likely due to an increased susceptibility of $\mathrm{Sn}^{2+}$ to oxidation. This is also corroborated by electroluminescence measurements from full devices (Figure S28), for which we observe diminishing EL signals beyond $50 \%$ Sn content.

Given that both $30 \%$ and $50 \%$ Sn compositions fall within the compositional zone of good optoelectronic quality and display key band gaps that are respectively ideal for single-junction devices or the bottom sub-cell in all-perovskite multi-junctions (Figure 1a and Figure S4), they were natural choices for further optimisation. Prior to this point, we have intentionally used the same set of fabrication parameters across the entire range of $\mathrm{Pb}: \mathrm{Sn}$ compositions in order to make valid comparisons. Now, we independently fine-tune the processing parameters for the $30 \%$ and $50 \%$ Sn devices in an optimisation effort to specifically target improved photovoltaic performance. In both cases, we replace the FTO transparent electrode with indium tin oxide (ITO), which reduces parasitic absorption and reflection losses (see Figure S29). For the 50\% composition, we replace the bilayer polymer p-type stack with PEDOT:PSS, which is almost exclusively used for low band gap solar cells. ${ }^{15,19,26-28}$ For the $30 \%$ Sn composition, we reduced the $\mathrm{Pb}(\mathrm{SCN})_{2}$ content from $6 \%$ molar excess to $5 \%$, which has a two-fold benefit of both slightly red-shifting the band gap from $1.35 \mathrm{eV}$ to the ideal value of $1.33 \mathrm{eV}$ and removing the $\mathrm{PbI}_{2}$ impurity phase from the XRD pattern (Figure S30). In Figure 5, we show that our champion $1.33 \mathrm{eV}$ and $1.30 \mathrm{eV}$ band gap solar cells achieve $17.6 \%$ and $18.0 \%$ efficiencies, respectively, in both the current-voltage characteristics (Figure 5a) and steady-state power output (Figure 5c) (see Table S5-S6 for the mean and champion photovoltaic performance metrics). We confirm the band gaps by taking the position of the derivative of the EQE spectrum (Figure 5d, Figure S31) and further corroborate with the EL spectra peaks (Figure S32). Although the most efficient narrow and ideal band gap perovskite solar cells to date have almost exclusively used at least $30 \% \mathrm{MA}^{+}$at the A-site, we demonstrate with our $1.30 \mathrm{eV}$ and $1.33 \mathrm{eV}$ champion devices that FA:Cs-based compositions can be competitive with best-inclass absorbers while also proffering the expected advantage of improved thermal stability. ${ }^{42}$ Similarly, the highest performing $1.35 \mathrm{eV}$ ideal band gap compositions reported to date both contain some amount of bromide, with efficiencies of $20.6 \%$ and $17.6 \%$ achieved respectively by $\left(\mathrm{FA}_{0.7} \mathrm{MA}_{0.3}\right)\left(\mathrm{Pb}_{0.7} \mathrm{Sn}_{0.3}\right) \mathrm{I}_{3}$ with a $12 \%$ addition of guanidinium bromide and $\mathrm{MA}\left(\mathrm{Pb}_{0.5} \mathrm{Sn}_{0.5}\right)\left(\mathrm{I}_{0.8} \mathrm{Br}_{0.2}\right)_{3}{ }^{40,66}$ While we currently match the efficiency of only one of these with our $1.33 \mathrm{eV}$ absorber, our choice of neat iodide at the $\mathrm{X}$-site instead of a mixed-halide system avoids any potential stability issues arising from light-induced halide segregation. ${ }^{67-70}$ 
M. Klug et al.

\section{Energy \&}

Environmental Science

Conclusion. We have assessed the mixed-metal $\mathrm{FA}_{0.83} \mathrm{Cs}_{0.17}\left(\mathrm{~Pb}_{1-y} \mathrm{Sn}_{\mathrm{y}}\right) \mathrm{I}_{3}$ perovskite system to evaluate how varying the Sn content impacts photovoltaic performance and optoelectronic quality in compositions spanning the full band gap range of 1.28 to $1.55 \mathrm{eV}$. We include in our study an examination of the impact of very low Sn contents $(<1 \%)$ on material and photovoltaic properties and whilst very small additions of Sn have negligible impact upon the optoelectronic properties of the perovskite, we find that exceeding $0.1 \%$ Sn replacement can introduce substantial defectiveness into the perovskite material. This is evidenced most dramatically by PL lifetimes and PLQE values exceeding $1 \mu$ s and 15\% respectively, when the nominal Sn content is less than $0.1 \%$, but dropping precipitously to $10 \mathrm{~ns}$ and effectively $0 \%$ when $\mathrm{Sn}$ comprises around $1 \%$ of the metal content. We identify the compositional range of $0.5-20 \% \mathrm{Sn}$ as being "optoelectronically defective" due to the dramatic reductions in photovoltaic performance and increases in non-radiative voltage loss that occur in this range, in comparison to their neat $\mathrm{Pb}$ and higher $\mathrm{Sn}$ content low band gap counterparts. For $\mathrm{Sn}$ content beyond $20 \%$, we show that the optoelectronic quality is restored, which we demonstrate with champion solar cells yielding steady-state photovoltaic efficiencies up to $18.0 \%$ for a low band gap composition $(50 \% \mathrm{Sn})$ and $17.6 \%$ for an ideal single-junction $1.33 \mathrm{eV}$ band gap absorber $(30 \%$ $\mathrm{Sn})$. Although $\mathrm{Pb}: \mathrm{Sn}$ materials are promising candidates for integration into both monolithic perovskite tandems and single-junction designs, compositions with band gaps between 1.35 and $1.5 \mathrm{eV}$, which may be desirable for the middle absorber layer in a triple-junction cell, are not feasible unless strategies are developed to overcome the inherent low optoelectronic quality we observe within this compositional range.

Future work is required to determine whether the poor optoelectronic quality we observed within the "defective zone" is intrinsic to this compositional range and will always be defective, or whether routes can be developed to improve material quality. Regardless of the outcome, the identification of the compositional "defective zone" already provides a clear and practical path to addressing the issue: simply avoid using $\left(\mathrm{FA}_{0.83} \mathrm{Cs}_{0.17}\right)\left(\mathrm{Pb}_{1-\mathrm{y}} \mathrm{Sn}_{\mathrm{y}}\right) \mathrm{I}_{3}$ perovskites with bandgaps between 1.35 and $1.5 \mathrm{eV}$, in both single-junction and multi-junction designs. For example, replacing the $1.44 \mathrm{eV}$ absorber at the middle junction of an "ideal" perovskiteperovskite-silicon triple-junction with a $1.35 \mathrm{eV}$ absorber, which can exist just outside the "defective zone," would only slightly reduce the predicted power conversion efficiency by about one absolute percentage point for realistic device architectures. ${ }^{6}$ Alternatively, efforts could focus on an all-perovskite triple-junction configuration, which avoids the problematic compositions entirely. ${ }^{6}$ This strategy of avoidance would circumvent the need for further 
M. Klug et al.

\section{Energy \&}

Environmental Science

absorber development and allow the materials presented herein to be immediately implemented into multi-junction devices, with perhaps only a negligible reduction in achievable efficiency.

Author Contributions. The manuscript was written through contributions of all authors. All authors have given approval to the final version of the manuscript. M.T.K. and H.J.S. conceived the idea and designed the experiments. M.T.K., T.G., L.L., and M.D.F. fabricated solar cells and samples for materials characterisation. Current-voltage characteristics and EQE measurements for solar cells were performed by M.T.K., J.M.B., and J.B.P. Optical-pump terahertz-probe measurements and analysis performed by R.L.M. Steady-state photoluminescence spectra measured by R.L.M. and M.T.K. X-ray photoelectron spectroscopy was performed by A.J.R. Photothermal deflection spectroscopy performed by S.M. Fouriertransform photocurrent spectroscopy performed by J.B.P. Film thicknesses, absorbance spectra, and time-resolved photoluminescence decay profiles were measured by M.T.K. Scanning electron microscopy were performed by M.T.K. Four-point probe conductivity measurements were performed and analysed by T.G. X-ray diffraction data was fit and analysed by H.C.S. Microstrain analysis was performed by M.T.K and Z.W. Electroluminescence measurements performed by M.T.K. and B.W. The project was supervised by H.J.S., L.M.H., M.B.J, and A.P.

Competing Financial Interest. Henry Snaith is a co-founder and Chief Science Officer of Oxford Photovoltaics, Ltd.

Acknowledgements. This work was supported by the UK Engineering and Physical Sciences Research Council (EPSRC) grant EP/P006329/1, EP/P033229/1 and EP/S004947/1. This work received funding from the European Union's Horizon 2020 research and innovation programme under the PerTPV project, grant agreement No 763977. L.M.H. and M.B.J. thank the Humboldt Foundation for Support through an Award. M.D.F. would like to acknowledge funding from the Engineering and Physical Sciences Research Council (EPSRC) EPSRC Centre for Doctoral Training in Plastic Electronic Materials (PE-CDT).

Electronic Supplementary Information (ESI) available. Contains details of materials and experimental methods, scanning electron micrographs, absorbance and steady-state photoluminescence spectra, Tauc plots of absorption spectra, X-ray photoelectron spectra, external quantum efficiency spectra and analysis, powder X-ray diffractograms, spectral mismatch calculation and mismatch factors, current-voltage characteristics and steady-state power time traces for representative devices, tables of mean and champion photovoltaic 
M. Klug et al.

\section{Energy \&}

Environmental Science

performance metrics, details of the charge carrier mobility calculation details, photoluminescence quantum efficiency spectra, time-resolved photoluminescence lifetime calculation details, a discussion on photothermal deflection spectroscopy, Williamson-Hall analysis, Urbach tails measured with Fourier-transform photocurrent spectroscopy, peak fits for photoluminescence and external quantum efficiency derivatives, and electroluminescence spectra.

\section{References.}

1 H. J. Snaith, Nat. Mater., 2018, 17, 372.

2 A. Abate, J.-P. Correa-Baena, M. Saliba, M. S. Su'ait and F. Bella, Chem. - Eur. J., 2018, 24, 30833100 .

3 Y. Rong, Y. Hu, A. Mei, H. Tan, M. I. Saidaminov, S. I. Seok, M. D. McGehee, E. H. Sargent and H. Han, Science, 2018, 361, eaat8235.

4 S. D. Stranks and H. J. Snaith, Nat. Nanotechnol., 2015, 10, 391-402.

5 J. Werner, B. Niesen and C. Ballif, Adv. Mater. Interfaces, 2017, 5, 1700731.

6 M. T. Hörantner, T. Leijtens, M. E. Ziffer, G. E. Eperon, M. G. Christoforo, M. D. McGehee and H. J. Snaith, ACS Energy Lett., 2017, 2, 2506-2513.

7 G. E. Eperon, S. D. Stranks, C. Menelaou, M. B. Johnston, L. M. Herz and H. J. Snaith, Energy Environ. Sci., 2014, 7, 982.

8 G. E. Eperon, G. M. Paterno, R. J. Sutton, A. Zampetti, A. A. Haghighirad, F. Cacialli and H. J. Snaith, J Mater Chem A, 2015, 3, 19688-19695.

9 S. A. Kulkarni, T. Baikie, P. P. Boix, N. Yantara, N. Mathews and S. Mhaisalkar, J Mater Chem A, 2014, 2, 9221-9225.

10 J. H. Noh, S. H. Im, J. H. Heo, T. N. Mandal and S. I. Seok, Nano Lett., 2013, 130321112645008.

11 R. J. Sutton, G. E. Eperon, L. Miranda, E. S. Parrott, B. A. Kamino, J. B. Patel, M. T. Hörantner, M. B. Johnston, A. A. Haghighirad, D. T. Moore and H. J. Snaith, Adv. Energy Mater., 2016, 1502458.

12 Q. A. Akkerman, V. D’Innocenzo, S. Accornero, A. Scarpellini, A. Petrozza, M. Prato and L. Manna, J. Am. Chem. Soc., 2015, 137, 10276-10281.

13 F. Hao, C. C. Stoumpos, R. P. H. Chang and M. G. Kanatzidis, J. Am. Chem. Soc., 2014, 136, 80948099.

14 W. Liao, D. Zhao, Y. Yu, N. Shrestha, K. Ghimire, C. R. Grice, C. Wang, Y. Xiao, A. J. Cimaroli, R. J. Ellingson, N. J. Podraza, K. Zhu, R.-G. Xiong and Y. Yan, J. Am. Chem. Soc., , DOI:10.1021/jacs.6b08337.

15 D. Zhao, Y. Yu, C. Wang, W. Liao, N. Shrestha, C. R. Grice, A. J. Cimaroli, L. Guan, R. J. Ellingson, K. Zhu, X. Zhao, R.-G. Xiong and Y. Yan, Nat. Energy, 2017, 2, 17018.

16 N. K. Noel, S. D. Stranks, A. Abate, C. Wehrenfennig, S. Guarnera, A. Haghighirad, A. Sadhanala, G. E. Eperon, S. K. Pathak, M. B. Johnston, A. Petrozza, L. Herz and H. Snaith, Energy Environ. Sci., , DOI:10.1039/c4ee01076k.

17 C. C. Stoumpos, C. D. Malliakas and M. G. Kanatzidis, Inorg. Chem., 2013, 52, 9019-9038.

18 F. Zuo, S. T. Williams, P.-W. Liang, C.-C. Chueh, C.-Y. Liao and A. K.-Y. Jen, Adv. Mater., 2014, 26, 6454-6460.

19 G. E. Eperon, T. Leijtens, K. A. Bush, R. Prasanna, T. Green, J. T.-W. Wang, D. P. McMeekin, G. Volonakis, R. L. Milot, R. May, A. Palmstrom, D. J. Slotcavage, R. A. Belisle, J. B. Patel, E. S. Parrott, R. J. Sutton, W. Ma, F. Moghadam, B. Conings, A. Babayigit, H.-G. Boyen, S. Bent, F. Giustino, L. M. Herz, M. B. Johnston, M. D. McGehee and H. J. Snaith, Science, , DOI:10.1126/science.aaf9717. 
M. Klug et al.

\section{Energy \&}

Environmental Science

20 Parrott Elizabeth S., Green Thomas, Milot Rebecca L., Johnston Michael B., Snaith Henry J. and Herz Laura M., Adv. Funct. Mater., 0, 1802803.

21 M. Anaya, J. P. Correa-Baena, G. Lozano, M. Saliba, P. Anguita, B. Roose, A. Abate, U. Steiner, M. Grätzel, M. E. Calvo, A. Hagfeldt and H. Míguez, J. Mater. Chem. A, 2016, 4, 11214-11221.

22 C. Liu, J. Fan, H. Li, C. Zhang and Y. Mai, Sci. Rep., 2016, 6, 35705.

23 R. Prasanna, A. Gold-Parker, T. Leijtens, B. Conings, A. Babayigit, H.-G. Boyen, M. F. Toney and M. D. McGehee, J. Am. Chem. Soc., 2017, 139, 11117-11124.

24 G. Kapil, T. S. Ripolles, K. Hamada, Y. Ogomi, T. Bessho, T. Kinoshita, J. Chantana, K. Yoshino, Q. Shen, T. Toyoda, T. Minemoto, T. N. Murakami, H. Segawa and S. Hayase, Nano Lett., 2018, 18, 3600-3607.

25 A. Rajagopal, P.-W. Liang, C.-C. Chueh, Z. Yang and A. K.-Y. Jen, ACS Energy Lett., 2017, 2, $2531-$ 2539.

26 G. Kapil, T. Bessho, C. H. Ng, K. Hamada, M. Pandey, M. A. Kamarudin, D. Hirotani, T. Kinoshita, T. Minemoto, Q. Shen, T. Toyoda, T. N. Murakami, H. Segawa and S. Hayase, ACS Energy Lett., 2019, 4, 1991-1998.

27 R. Lin, K. Xiao, Z. Qin, Q. Han, C. Zhang, M. Wei, M. I. Saidaminov, Y. Gao, J. Xu, M. Xiao, A. Li, J. Zhu, E. H. Sargent and H. Tan, Nat. Energy, 2019, 1-10.

28 J. Tong, Z. Song, D. H. Kim, X. Chen, C. Chen, A. F. Palmstrom, P. F. Ndione, M. O. Reese, S. P. Dunfield, O. G. Reid, J. Liu, F. Zhang, S. P. Harvey, Z. Li, S. T. Christensen, G. Teeter, D. Zhao, M. M. Al-Jassim, M. F. A. M. van Hest, M. C. Beard, S. E. Shaheen, J. J. Berry, Y. Yan and K. Zhu, Science, 2019, 364, 475.

29 J. Werner, T. Moot, T. A. Gossett, I. E. Gould, A. F. Palmstrom, E. J. Wolf, C. C. Boyd, M. F. A. M. van Hest, J. M. Luther, J. J. Berry and M. D. McGehee, ACS Energy Lett., 2020, 1215-1223.

30 M. T. Klug, A. Osherov, A. A. Haghighirad, S. D. Stranks, P. R. Brown, S. Bai, J. T.-W. Wang, X. Dang, V. Bulovic, H. J. Snaith and A. M. Belcher, Energy Env. Sci, 2017, 10, 236-246.

31 M. Konstantakou and T. Stergiopoulos, J. Mater. Chem. A, 2017, 5, 11518-11549.

32 R. L. Milot, M. T. Klug, C. L. Davies, Z. Wang, H. Kraus, H. J. Snaith, M. B. Johnston and L. M. Herz, Adv. Mater., 0, 1804506.

33 T. Leijtens, R. Prasanna, A. Gold-Parker, M. F. Toney and M. D. McGehee, ACS Energy Lett., 2017, 2, 2159-2165.

34 Y. Takahashi, H. Hasegawa, Y. Takahashi and T. Inabe, J. Solid State Chem., 2013, 205, 39-43.

35 W. Li, J. Li, J. Li, J. Fan, Y. Mai and L. Wang, J. Mater. Chem. A, 2016, 4, 17104-17110.

36 X. Xu, C.-C. Chueh, P. Jing, Z. Yang, X. Shi, T. Zhao, L. Y. Lin and A. K.-Y. Jen, Adv. Funct. Mater., 2017, 27, 1701053.

37 I. Chung, B. Lee, J. He, R. P. H. Chang and M. G. Kanatzidis, Nature, 2012, 485, 486-489.

38 M. H. Kumar, S. Dharani, W. L. Leong, P. P. Boix, R. R. Prabhakar, T. Baikie, C. Shi, H. Ding, R. Ramesh, M. Asta, M. Graetzel, S. G. Mhaisalkar and N. Mathews, Adv. Mater., 2014, 26, 7122-7127.

39 B. Zhao, M. Abdi-Jalebi, M. Tabachnyk, H. Glass, V. S. Kamboj, W. Nie, A. J. Pearson, Y. Puttisong, K. C. Gödel, H. E. Beere, D. A. Ritchie, A. D. Mohite, S. E. Dutton, R. H. Friend and A. Sadhanala, Adv. Mater., 2017, 29, 1604744.

40 X. Zhou, L. Zhang, X. Wang, C. Liu, S. Chen, M. Zhang, X. Li, W. Yi and B. Xu, Adv. Mater., 2020, n/a, 1908107.

41 D. P. McMeekin, G. Sadoughi, W. Rehman, G. E. Eperon, M. Saliba, M. T. HÃ $\mid$ rantner, A.

Haghighirad, N. Sakai, L. Korte, B. Rech, M. B. Johnston, L. M. Herz and H. J. Snaith, Science, 2016, 351, 151-155.

42 T. Leijtens, R. Prasanna, K. A. Bush, G. E. Eperon, J. A. Raiford, A. Gold-Parker, E. J. Wolf, S. A. Swifter, C. C. Boyd, H.-P. Wang, M. F. Toney, S. F. Bent and M. D. McGehee, Sustain. Energy Fuels, 2018, 2, 2450-2459.

43 W. Ke, C. Xiao, C. Wang, B. Saparov, H.-S. Duan, D. Zhao, Z. Xiao, P. Schulz, S. P. Harvey, W. Liao, W. Meng, Y. Yu, A. J. Cimaroli, C.-S. Jiang, K. Zhu, M. Al-Jassim, G. Fang, D. B. Mitzi and Y. Yan, Adv. Mater., 2016, 28, 5214-5221. 
M. Klug et al.

\section{Energy \&}

Environmental Science

44 S. Yang, W. Liu, L. Zuo, X. Zhang, T. Ye, J. Chen, C.-Z. Li, G. Wu and H. Chen, J. Mater. Chem. A, 2016, 4, 9430-9436.

45 Y. Yu, C. Wang, C. R. Grice, N. Shrestha, J. Chen, D. Zhao, W. Liao, A. J. Cimaroli, P. J. Roland, R. J. Ellingson and Y. Yan, ChemSusChem, 2016, 9, 3288-3297.

46 U. Rau, B. Blank, T. C. M. Müller and T. Kirchartz, Phys. Rev. Appl., 2017, 7, 044016.

47 P. K. Nayak, S. Mahesh, H. J. Snaith and D. Cahen, Nat. Rev. Mater., 2019, 4, 269-285.

48 W. Travis, E. N. K. Glover, H. Bronstein, D. O. Scanlon and R. G. Palgrave, Chem Sci, 2016, 7, 45484556.

49 J. Lee, H. Kang, G. Kim, H. Back, J. Kim, S. Hong, B. Park, E. Lee and K. Lee, Adv. Mater., 2017, 29, 1606363.

50 M. Stolterfoht, C. M. Wolff, J. A. Márquez, S. Zhang, C. J. Hages, D. Rothhardt, S. Albrecht, P. L. Burn, P. Meredith, T. Unold and D. Neher, Nat. Energy, 2018, 3, 847-854.

51 H. J. Snaith, Energy Environ. Sci., 2012, 5, 6513-6520.

52 E. Mosconi, P. Umari and F. De Angelis, J Mater Chem A, 2015, 3, 9208-9215.

53 R. L. Milot, G. E. Eperon, T. Green, H. J. Snaith, M. B. Johnston and L. M. Herz, J. Phys. Chem. Lett., 2016, 7, 4178-4184.

54 A. R. Bowman, M. T. Klug, T. A. S. Doherty, M. D. Farrar, S. P. Senanayak, B. Wenger, G. Divitini, E. P. Booker, Z. Andaji-Garmaroudi, S. Macpherson, E. Ruggeri, H. Sirringhaus, H. J. Snaith and S.

D. Stranks, ACS Energy Lett., 2019, 4, 2301-2307.

55 L. M. Herz, ACS Energy Lett., 2017, 2, 1539-1548.

56 W. Rehman, R. L. Milot, G. E. Eperon, C. Wehrenfennig, J. L. Boland, H. J. Snaith, M. B. Johnston and L. M. Herz, Adv. Mater., 2015, 27, 7938-7944.

57 W. Rehman, D. P. McMeekin, J. B. Patel, R. L. Milot, M. B. Johnston, H. J. Snaith and L. M. Herz, Energy Env. Sci, 2017, 10, 361-369.

58 F. Urbach, Phys Rev, 1953, 92, 1324-1324.

59 S. John, C. Soukoulis, M. H. Cohen and E. N. Economou, Phys Rev Lett, 1986, 57, 1777-1780.

60 J. Jean, T. S. Mahony, D. Bozyigit, M. Sponseller, J. Holovský, M. G. Bawendi and V. Bulović, ACS Energy Lett., 2017, 2, 2616-2624.

61 U. Rau, Phys. Rev. B, 2007, 76, 085303.

62 S. D. Stranks, ACS Energy Lett., 2017, 2, 1515-1525.

63 O. D. Miller, E. Yablonovitch and S. R. Kurtz, IEEE J. Photovolt., 2012, 2, 303-311.

64 M. R. Filip, G. E. Eperon, H. J. Snaith and F. Giustino, Nat. Commun., 2014, 5, 5757.

65 J.-H. Lee, N. C. Bristowe, J. H. Lee, S.-H. Lee, P. D. Bristowe, A. K. Cheetham and H. M. Jang, Chem. Mater., 2016, 28, 4259-4266.

66 Z. Yang, A. Rajagopal and A. K.-Y. Jen, Adv. Mater., 2017, 29, 1704418.

67 E. T. Hoke, D. J. Slotcavage, E. R. Dohner, A. R. Bowring, H. I. Karunadasa and M. D. McGehee, Chem Sci, 2015, 6, 613-617.

68 D. J. Slotcavage, H. I. Karunadasa and M. D. McGehee, ACS Energy Lett., 2016, 1, 1199-1205.

69 M. C. Brennan, S. Draguta, P. V. Kamat and M. Kuno, ACS Energy Lett., 2018, 3, 204-213.

70 S. Draguta, O. Sharia, S. J. Yoon, M. C. Brennan, Y. V. Morozov, J. S. Manser, P. V. Kamat, W. F. Schneider and M. Kuno, Nat. Commun., 2017, 8, 200. 
Figures.

a)
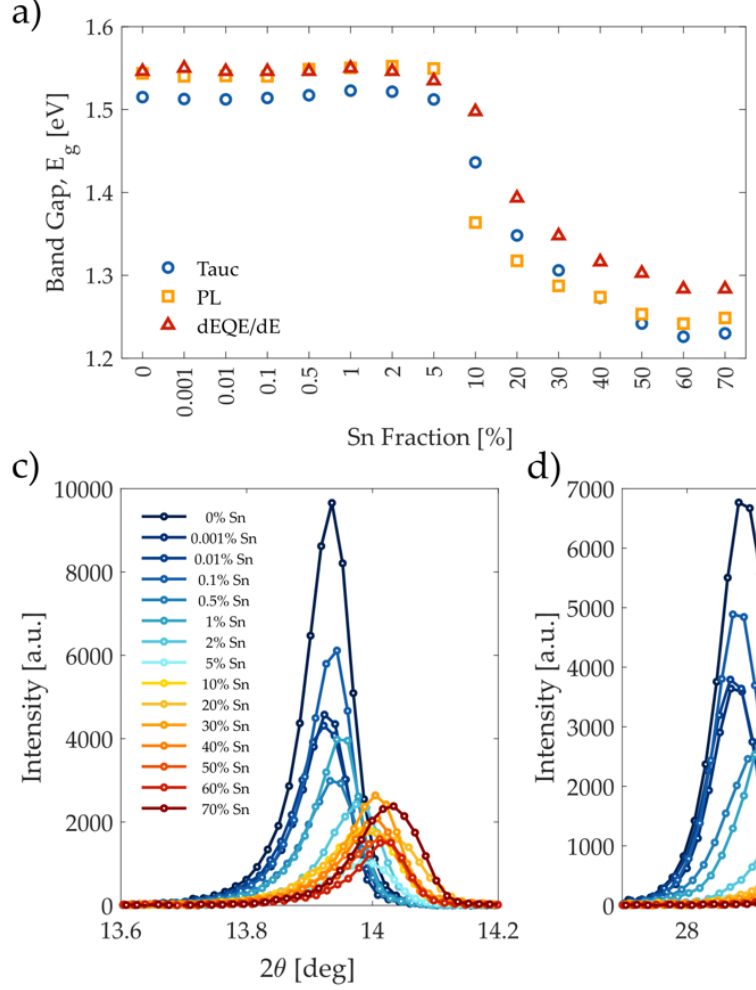

b)

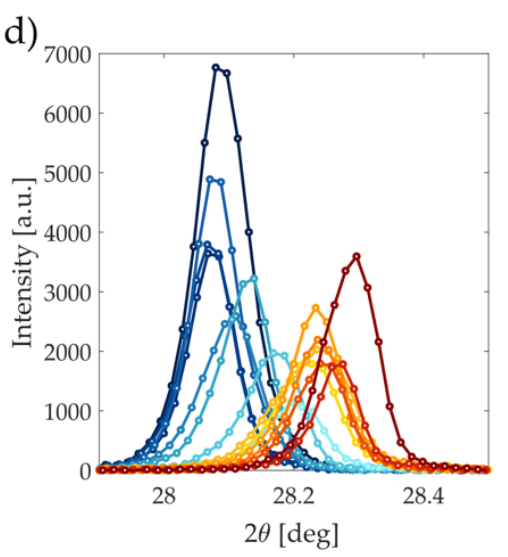

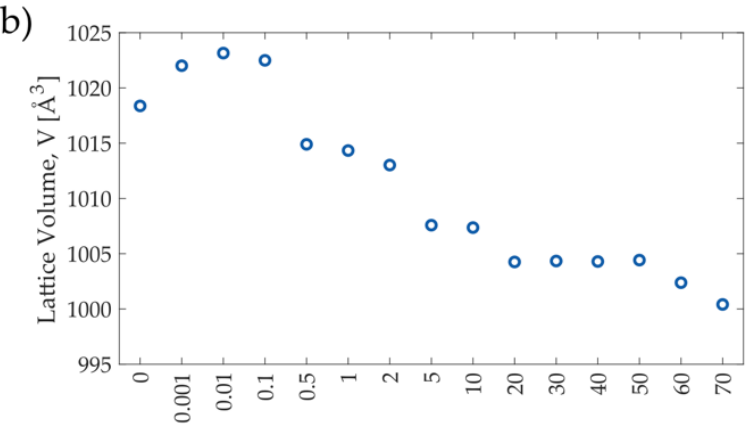

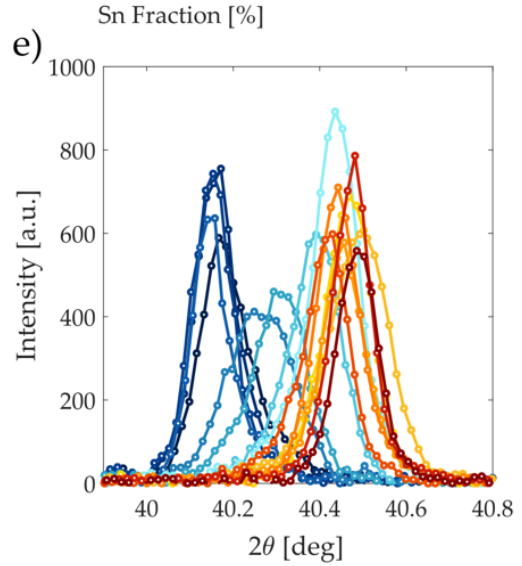

Figure 1. (a) Band gap as determined by the Tauc plot of UV-Vis-NIR absorption spectra, photoluminescence (PL) peak, and derivative of the external quantum efficiency (EQE) and (b) lattice volume as a function of $\mathrm{Sn}^{2+}$ fraction in thin-films of $\left(\mathrm{FA}_{0.83} \mathrm{Cs}_{0.17}\right)\left(\mathrm{Pb}_{1-\mathrm{y}} \mathrm{Sn}_{\mathrm{y}}\right) \mathrm{I}_{3}$. Local region around the (c) first, (d) second, and (e) third strongest X-ray diffraction peaks. The legend displayed in (c) also applies to (d) and (e).

a)

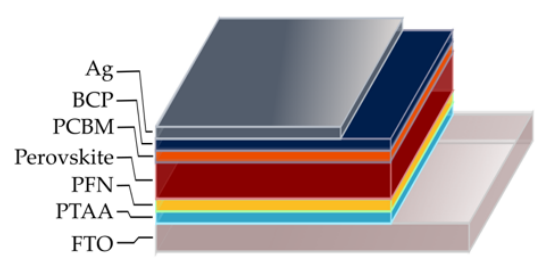

d)

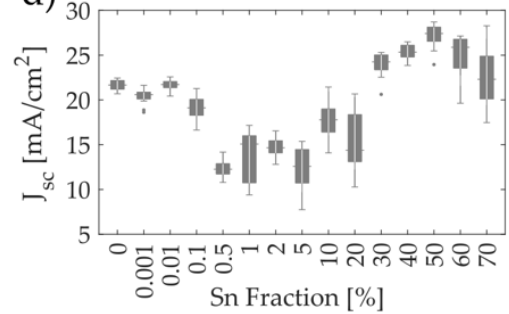

b)

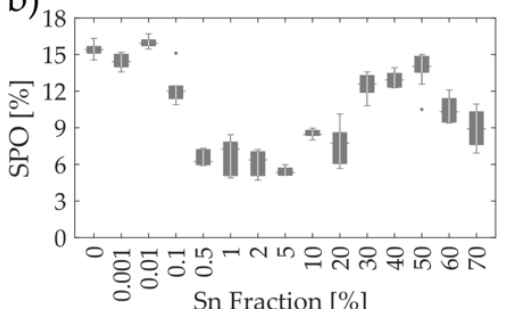

e)

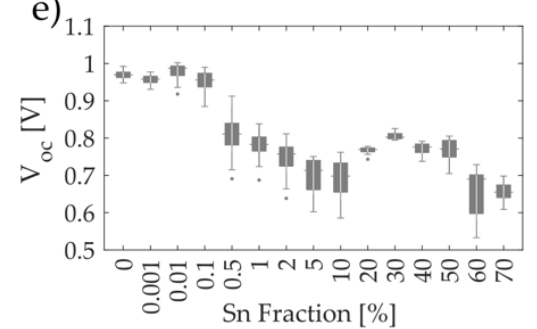

c)

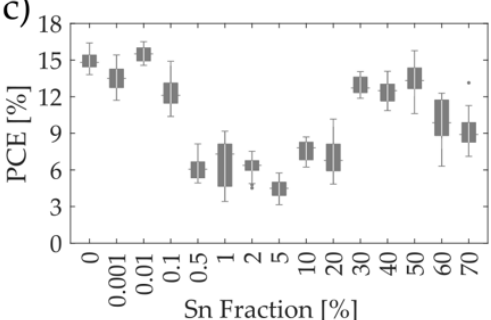

f)

Figure 2. (a) Device architecture of $\left(\mathrm{FA}_{0.83} \mathrm{Cs}_{0.17}\right)\left(\mathrm{Pb}_{1-\mathrm{y}} \mathrm{Sn}_{\mathrm{y}}\right) \mathrm{I}_{3}$ solar cells in the $\mathrm{p}-\mathrm{i}-\mathrm{n}$ configuration. Compositional dependence of (b) steady-state power output (SPO) and (c-f) photovoltaic performance metrics as determined from the current-voltage characteristics. Note that the short-circuit current density, $\mathrm{J}_{\mathrm{sc}}$, values have also been corrected for spectral mismatch to allow for valid comparison across compositions when illuminated with an equivalent 1 sun irradiance under the AM1.5G spectrum. 
M. Klug et al.

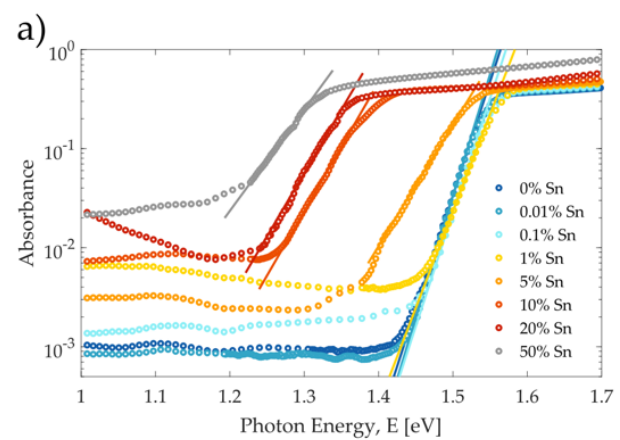

d)

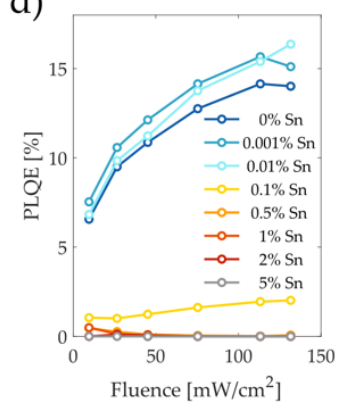

e) b)
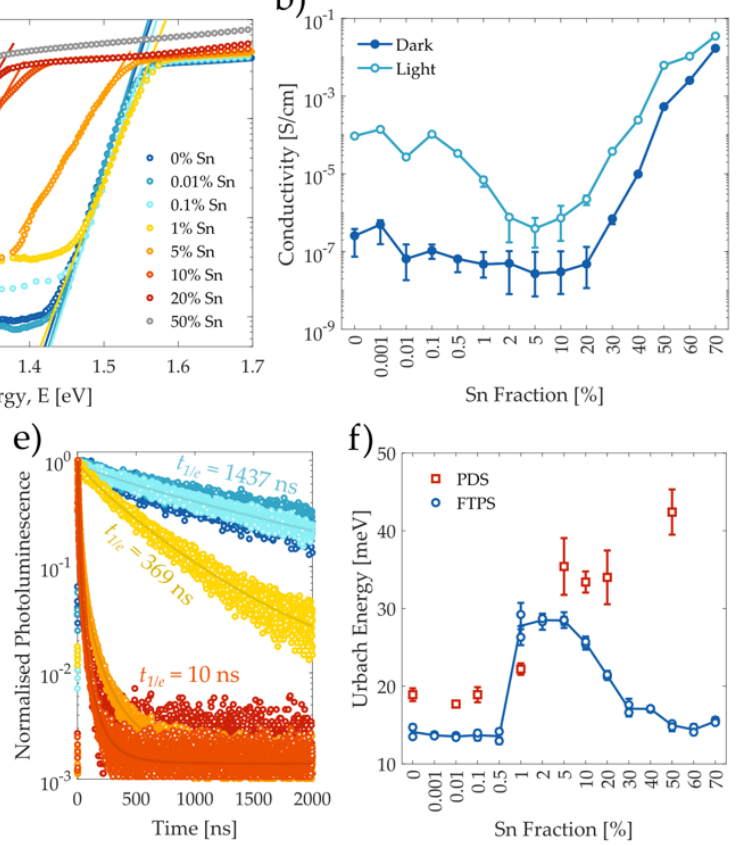

Energy \& Environmental Science

c)

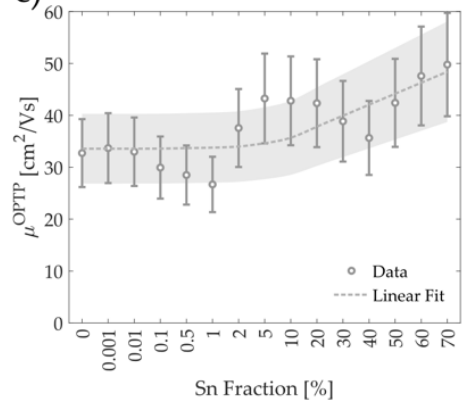

g)

Sn Fraction [\%]

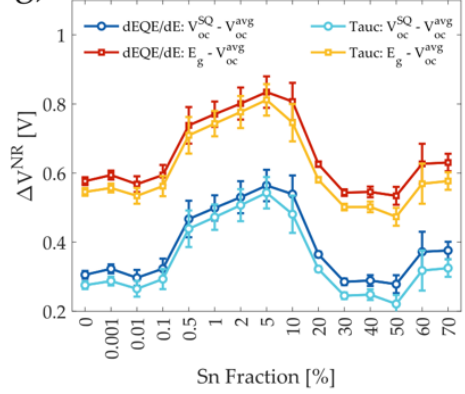

Figure 3. Optoelectronic characterisation of $\left(\mathrm{FA}_{0.83} \mathrm{Cs}_{0.17}\right)\left(\mathrm{Pb}_{1-\mathrm{y}} \mathrm{Sn}_{\mathrm{y}}\right) \mathrm{I}_{3}$ materials as a function of nominal Sn fraction. (a) Absorbance spectra measured through photothermal deflection spectroscopy (PDS) of perovskite thin-films. (b) Long-range conductivity measured with a four-point probe setup $(0.5 \mathrm{~mm}$ channel width) in the dark or under $\sim 0.1$ sun illumination. (c) Short-range charge carrier mobility determined by the optical-pump terahertz-probe (OPTP) measurement technique. Errors in charge carrier mobility values are estimated to be $\pm 20 \%$, which is the standard deviation in mobility values determined from a set of six nominally identical $\mathrm{FASnI}_{3}$ thin films. These were prepared similarly to the films in this study and are therefore considered representative of the expected sample-to-sample variation across this series. ${ }^{32}$ The dashed line corresponds to a linear fit to the data and the shaded region surrounding it represents the expected uncertainty. Please note the non-linear scale of the x-axis. (d) Photoluminescence quantum efficiency (PLQE) as a function of irradiance from a $532 \mathrm{~nm}$ laser and (e) photoluminescence decay profiles in encapsulated thin-films (circle markers) and the corresponding stretched-exponential fit (solid lines) - the legend displayed in (d) also applies to (e). (f) Urbach energy determined from neat thin-films with PDS or from full solar cells using Fourier-transform photocurrent spectroscopy (FTPS). (g) Mean non-radiative voltage loss, $\Delta V^{N R}$, as determined by the difference between either the measured mean open-circuit voltage, $V_{o c}^{a v g}$, and the band gap, $E_{g}$, (squares) or the theoretical maximum open-circuit voltage in the radiative limit, $V_{o c}^{S Q}$, (circles) using either the Tauc plot or dEQE/dE peak to define $E_{g}$. 
M. Klug et al.

a)

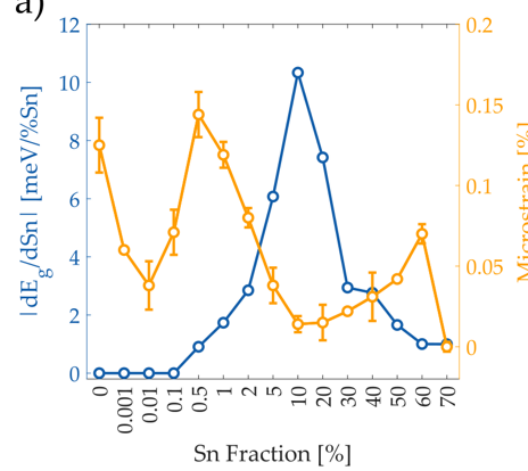

b)

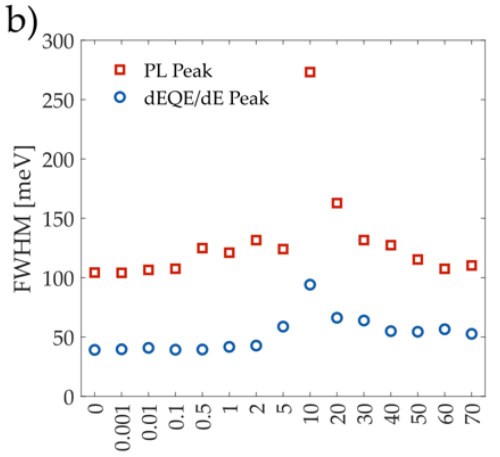

Sn Fraction [\%]
Energy \&

Environmental Science

c)

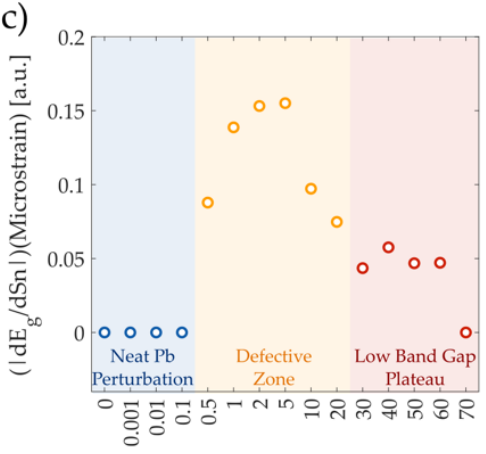

Sn Fraction [\%]

Figure 4. (a) Compositional derivative of the band gap, $\mathrm{dE}_{\mathrm{g}} / \mathrm{dSn}$, (left axis) and lattice microstrain (right axis), (b) photoluminesence (PL) and EQE derivative peak widths as defined by the full-width halfmaximum (FWHM) values, and (c) the product of the two quantities in panel (a) as a function of $\mathrm{Sn}$ content in $\left(\mathrm{FA}_{0.83} \mathrm{Cs}_{0.17}\right)\left(\mathrm{Pb}_{1-\mathrm{y}} \mathrm{Sn}_{\mathrm{y}}\right) \mathrm{I}_{3}$ thin-films.

a)

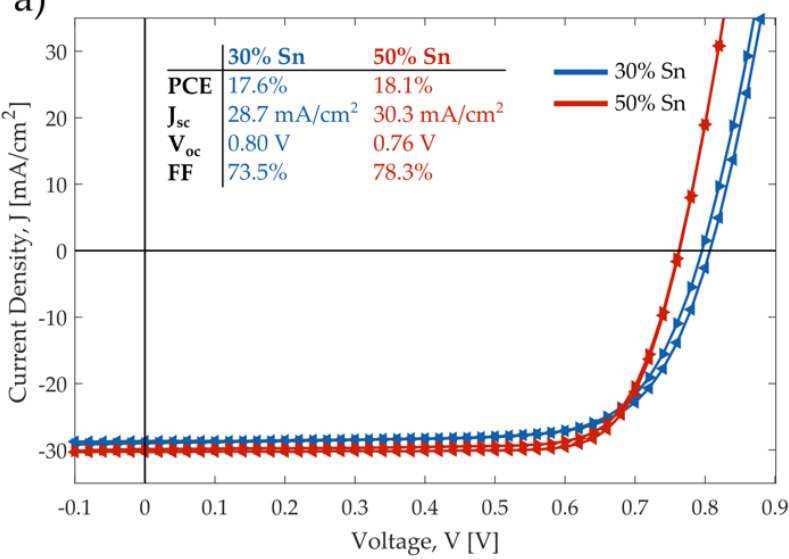

c)

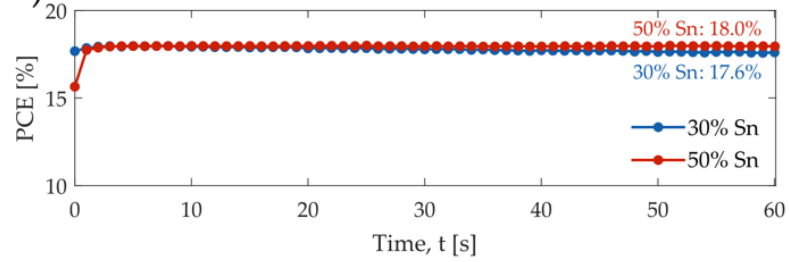

b)

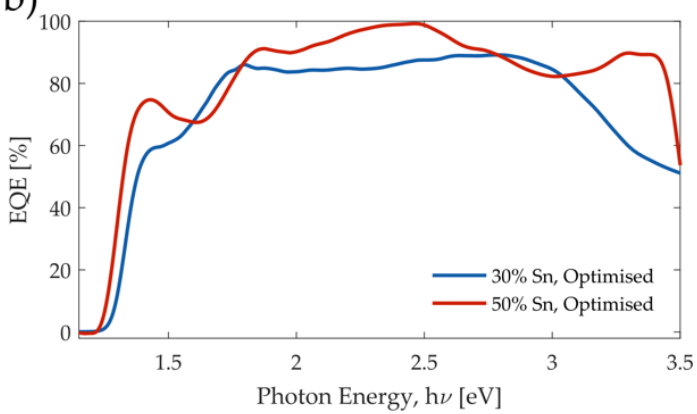

d)

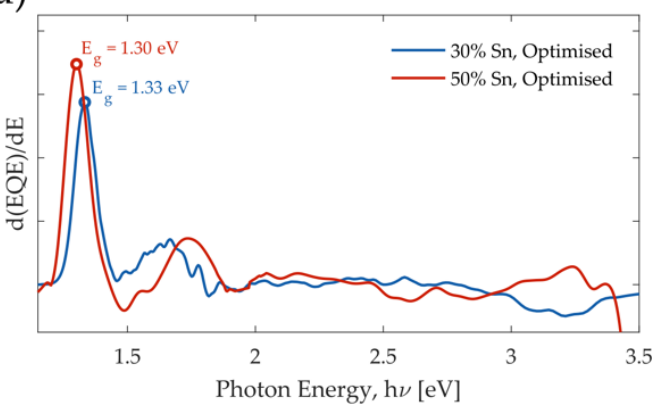

Figure 5. (a) Current-voltage characteristic for forward (right-pointing triangles) and reverse (leftpointing triangles) scans, (b) external quantum efficiency (EQE), (c) steady-state power output, and (d) derivative of $\mathrm{EQE}$ with respect to photon energy for the champion $1.33 \mathrm{eV}$ band gap $\left(\mathrm{FA}_{0.83} \mathrm{Cs}_{0.17}\right)\left(\mathrm{Pb}_{0.7} \mathrm{Sn}_{0.3}\right) \mathrm{I}_{3}$ and $1.30 \mathrm{eV}\left(\mathrm{FA}_{0.83} \mathrm{Cs}_{0.17}\right)\left(\mathrm{Pb}_{0.5} \mathrm{Sn}_{0.5}\right) \mathrm{I}_{3}$ solar cells. The power conversion efficiency values have been corrected to account for spectral mismatch. The $1.33 \mathrm{eV}$ and $1.30 \mathrm{eV}$ solar cells were illuminated with an equivalent 0.955 and 1.000 suns, respectively. 